\title{
Sign Language to Number by Neural Network
}

\author{
Shekhar Singh \\ Assistant Professor \\ CSE, Department \\ PIET, samalkha, Panipat, India
}

\author{
Pradeep Bharti \\ Assistant Professor \\ CSE, Department \\ PIET, samalkha, Panipat, India
}

\author{
Deepak Kumar \\ Lecturer \\ CSE, Department \\ PIET, samalkha, Panipat, India
}

\begin{abstract}
In this paper is presented an automatic deaf language to number recognition system. Sign language number recognition system lays down foundation for hand shape recognition which addresses real and current problems in signing in the deaf community and leads to practical applications. The scheme is based on neural network (NN) classifier using a back propagation. The input for the sign language number recognition system is 1000 Indian Sign Language number images with $640 \times 480$ pixels size. The input parameter vector to neural network is the Fisher score, which represents the derivate of the matrix of symbol probability in hidden Markov model (HMM). The HMM, which needs a sequence to be trained and used, is fed by the hand contour chain code. Besides, an improvement on the calculation of Fisher score is introduced by means of reducing the kernel scores variance. The error ratio classifying hand number of the proposed system is less than $1.4 \%$ with our database. The system learns and recognizes the Indian Sign Language number in training and testing phase using Hidden Markov Model and neural network. The system uses neural network for training and testing phase. The sign language recognizer could recognize Indian sign language number with $98.52 \%$ accuracy.
\end{abstract}

\section{Keywords}

Computer vision, Human computer interaction, Sign language, Image processing, Chain Code, HMM, Fisher score, Neural network, Sign language recognition.

\section{INTRODUCTION}

The growing commitment of the society in reducing the barriers to persons with disabilities, added to the advances of the computers and form recognition methods, has motivated the development of the present system that transforms the sign language in the Indian number, because in each country the sign language is different. Sign language is local, in contrast with the general opinion which assumes it is universal. Different countries and at times even regions within a country have their own sign languages. Sign language is a natural language for the deaf. Most hearing people do not understand any sign language and know very little about deafness in general. Although many deaf people lead successful and productive lives, this communication barrier can have problematic effects on many aspects of their lives. There are main categories in sign language recognition, namely hand shape classification and isolated sign language recognition.

This system bases its operation in providing in the input an image of a sign number Indian hand sign, and in their output the number of the number system that corresponds it, just as it is observed in the following figure, including the $\mathrm{N}$ Indian number.

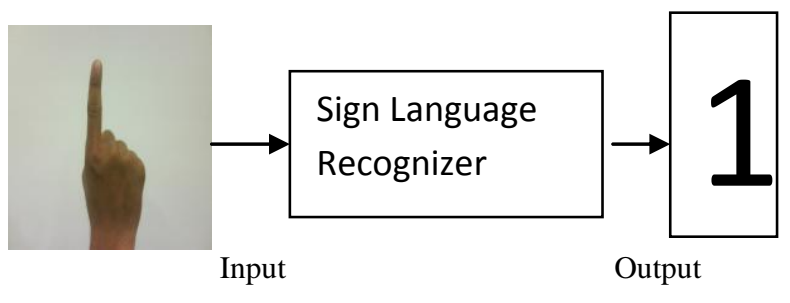

Figure 1: Translation from sign alphabet automatic recognition system, until the number

The used procedure in this automatic recognition is based in the adequate modeled of the hand sign by Neural Network. For it, it has been realized the calculation of Fisher score, which is extracted of HMM [3]. This HMM is fed with the chain code, determined from the band sign image. For this process, the first step has been arranged the capture of samples. It is necessary to create a database with hand sign pictures of the sign number of numerous persons, being the ideal infinite. In our case, it was enclosed for fifty different people (100 hand signs each one). Subsequently, it has been applied a image preprocessing, transforming the color image in a white and black one, with more reduced size, which defines the hand outline.

With this outline, we are going to extract a series of parameter, associated in a vector that defines the contour of the hand sign with chain code [4] [6], and it is classified and parameterized by HMM. From these HMM, some parameters are going to extract, based on the emission probability of the vectors, which are going to determine the score Fisher [1], for classifying it with Neural Network. This system will he supervised with a training process, in which is learned to differentiate some hand signs of other; and a other test process, where the models will be verified. This process is resumed in the following figure 1 . The continued structure in this article is the one that continues. In the second section the creation of the database. In the third, the applied image processing. Subsequently, the calculation of Fisher score by HMM. In the fifth section the neural network classifier. The sixth sections are all the realized experiments and finally the result analysis, conclusions and references.

\section{RELATED LITERATURE}

A vision-based medium Chinese sign language recognition system [9] is developed using tied mixture density hidden Markov models. In this system, the recognition vocabulary contains 439 CSL signs, including 223 two-handed and 216 one-handed signs. Their experimental results show that the proposed methods could achieve an average recognition accuracy of $92.5 \%$ on 439 signs, including $93.3 \%$ on twohanded signs and $91.7 \%$ on one-handed signs, respectively. 
In recent research of sign language recognition [10], a novel viewpoint invariant method for sign language recognition is proposed. The recognition task is converted to a verification task under the proposed method. This method verifies the uniqueness for a virtual stereo vision system, which is formed by the observation and template. The recognition vocabulary of this research contains 100 CSL signs. The image revolution is $320 \times 240$ pixels. The proposed method achieves an accuracy of $92 \%$ at rank 2 .

A framework for recognizing American Sign Language (ASL) is developed using hidden Markov model [12]. The data set consist of 499 sentences, between 2 and 7 signs long, and a total of 1604 signs from a 22 -sign vocabulary. They collect these data with an Ascension Technologies MotionStarTM system at 60 frames per second. In addition, they collect data from the right hand with a Virtual Technologies CybergloveTM, which records wrist yaw, pitch, and the joint and abduction angles of the fingers, also at 60 frames per second. The result shows clearly that the quadrilateral-based description of the hand shape $(95.21 \%)$ is far more robust than the raw joint angles $(83.15 \%)$. The best result is achieved using PaHMM monitoring movement channel right hand and hand shape right hand with $88.89 \%$ sentence accuracy and $96.15 \%$ word accuracy.

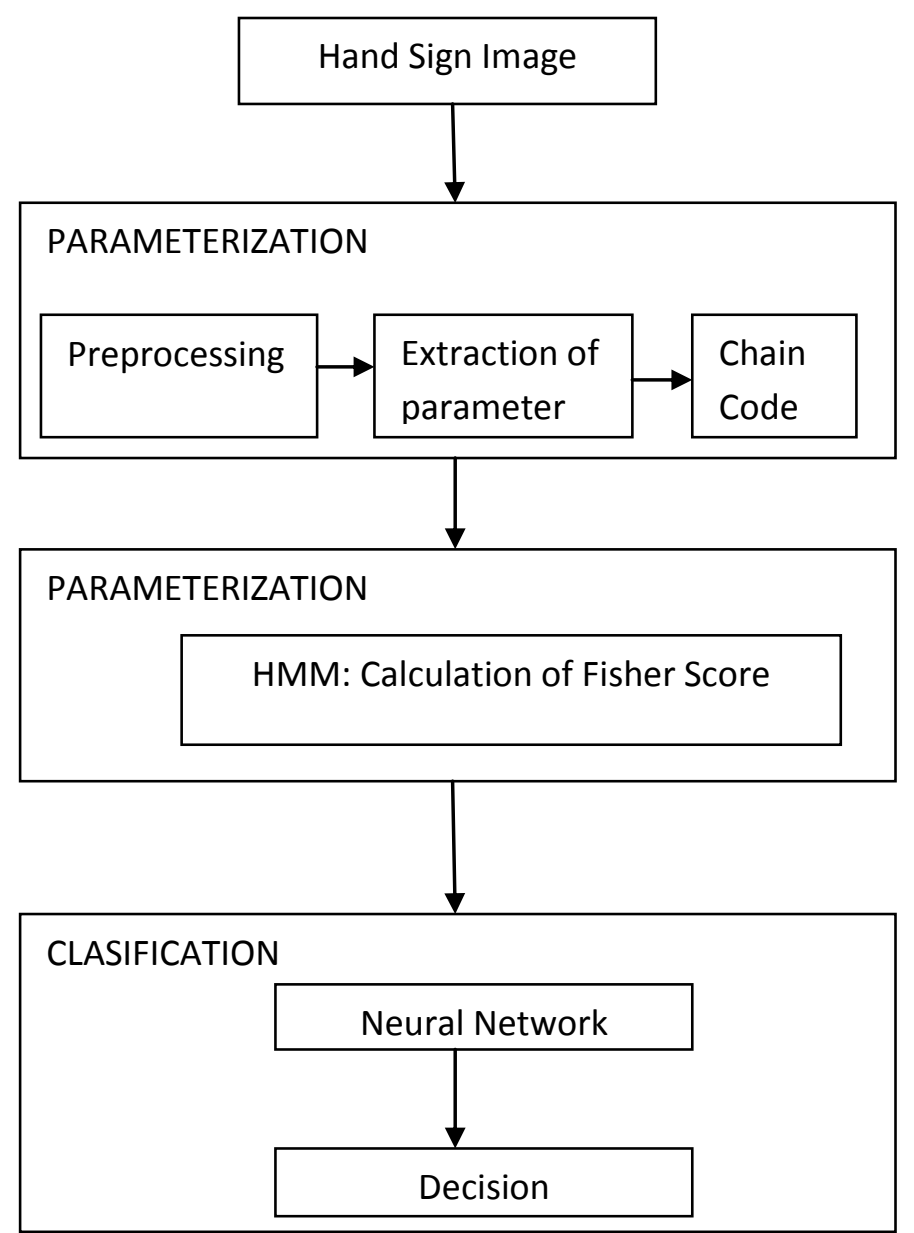

Figure 2: Procedure of automatic hand sign recognition system

\section{BUILDING OF DATABASE}

A database has been created for the execution of this paper that is enclosed of 100 hand signs or patterns, collecting 50 samples of each one all of different peoples. These images have been captured by a digital photograph camera. The creation of this database has had a series of difficulties, among them is emphasized the communications barrier of working with people with auditory disability. By another side, it has been worked with images static, without movement.

Some statistic data from database are the following:

- A $60 \%$ of males and a $40 \%$ of female.

- The $30 \%$ of the peoples are deaf and the remainder not.

- The distribution in ages varies since the 20 to the 60 years old.

- The images have a size of $640 \times 480$ pixels (wide $\mathrm{x}$ high), with a resolution 24 bits of color, in a format REG, that is to say, 8 bits for each one of the fundamental colors.

Finally, the hand signs are presented in the figure 3 , in particular, a sample of each one of the hand signs to recognize.

\section{SIGN IMAGE PRE-PROCESSING}

In this process are going to take the color images for transforming to binary images of hand sign shape (white and black) with a fixed height of 400 pixels, conserving the relation with respect the width.

The following steps are;

1. Filter RGB image for eliminating the noise, hue, saturation effects.

2. Convert RGB image in YCBCR color image by eliminating the hue and saturation.

3. Convert YCBCR image in grayscale by eliminating the hue and saturation.

4. Filter grayscale image for eliminating the noise, hue and saturation.

5. Enhance contrast using histogram equalization [4]. To realize this process, it is equalized the histogram of the different levels of gray, under a lineal function (see the figure 4), but without affecting to the darkest points, only for the clearest parts (the hand sign), marking differences among the shadow of the hand and the background; and the own hand.

6. Take the frame out to eliminate some border effects (see figure 5).

7. Convert image to binary image by Thresholding. The Thresholding is computed by means of Otsu's method, which chooses the threshold to minimize the interclass variance of the Thresholding black and white pixels. With this step is finished for determining the hand as an object.

8. Morphologic operators [5]. It is applied the dilatation operations in a first place, and after the erosion, as effect to recover the conditions of size of the image. The elimination of the noise is other desired effect. The dilatation is going to unite the holes that can remain in the line of the contour. Subsequently the hand is filled as object, and finally recovers the original dimension, by means of the erosion, that is able to eliminate the possible noises happened by the own photo.

9. Reduction. The size of image is reduced in one quarter, for reducing the size of the final vector.

10. Calculation of the contour [6] [7]. It is calculated the hand contour, which determines the hand sign on the background of the image, with the particularity that the 
connection among pixels is alone one pixel, with a connectivity among neighbors of 8 , that is chain code.

11. Adjustment of the wrist. The image is trimmed slightly with the intention to determine the side of the one that the hand sign arises. Adjustment of high of the image. Finally it is fixed the value of the height, maintaining the proportionality with regard to the width. In this way the information is not loosen, indistinctly that the hand sign is horizontal, vertical up or vertical downward.

12. In the figure 5 , this process of processing image is shown, in particular, for the hand sign of the number 1 .

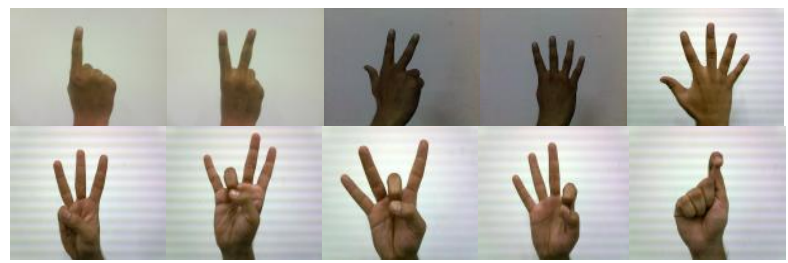

Figure 3: Hand signs that conform the database

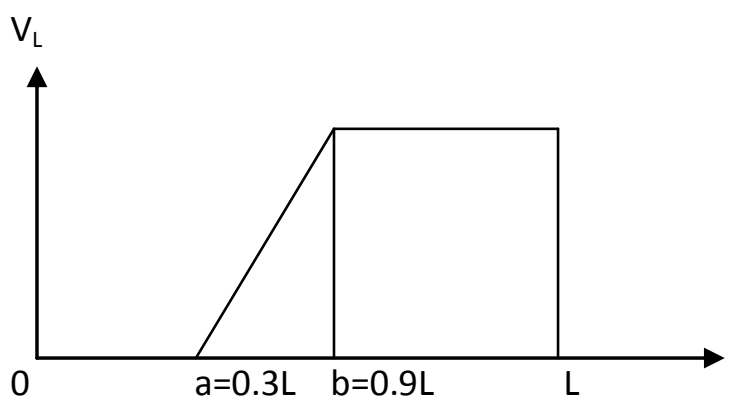

Figure 4: Applied equalization to the intensity

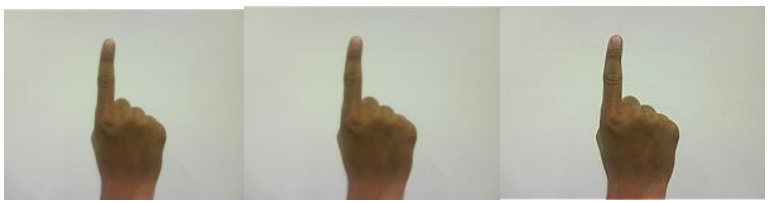

Original Image (a) Resize Image (b) Filter Image
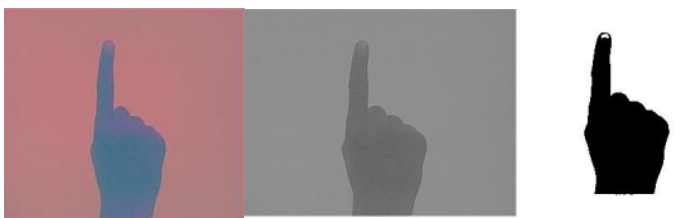

(c) YCBCR Color Image (d) Gray Image

(e) Binary Image
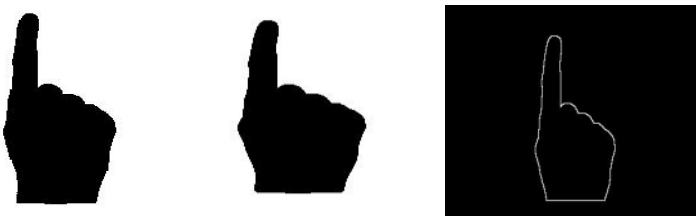

(f) Morphologic

(g) Close

(h) Outline
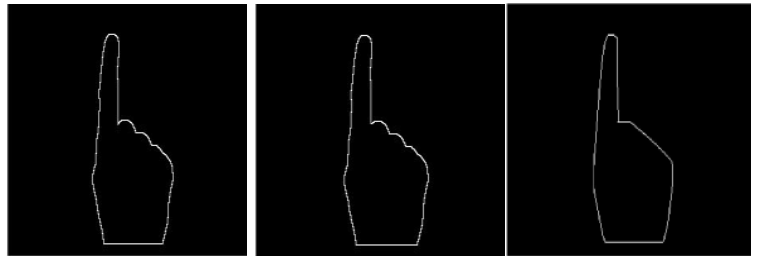

(i) Crop Image

(j) Adjustment

(k) minimum polygon adjustment

Figure 5: Steps of the applied processing image

\section{FISHER SCORE CALCULATION}

Once it is obtained all the outline images of the hand signs, it is realized the calculation of Fisher score. This process comprises in three steps;

- Extraction of parameter from outline: chain code [4].

- Creation HMM with chain code as input [6] [7].

- Calculation of Fisher score from gradient of logarithm of the observation symbol probability distribution [1] [3].

\subsection{Chain code from outline hand}

This vector of the contour hand sign is obtained with the mask of the figure 6 , observing the position of each pixel with their adjacent one. This vector is formed by numbers from the 1 to the 8 that describes the outline of the hand sign. The information that is extracted, describes the sequence of the hand, accompanied by temporary information, because all the hand signs are acquired in the same order and sense. This information is very important for the recognizer based on HMM, therefore utilizes this information to distinguish the different hand signs. It is fixed a criterion of start for the obtaining of this vector, verifying first if is a vertical, horizontal since down or vertical since up hand sign, for this is not rotation invariant. It is begun for seeking write pixels with the following order of priority: first, the first column of the left (horizontal hand sign), the last row (vertical since down) or the first row (vertical since up). This order of priority will depend; evidently, of the probability to be produced each type of hand sign, thus, the vertical hand signs since up are the last, since only there are two.

\begin{tabular}{|c|c|c|}
\hline 1 & 2 & 3 \\
\hline 8 & $\mathrm{X}$ & 4 \\
\hline 7 & 6 & 5 \\
\hline
\end{tabular}

Figure 6: Mask of composition of the vector of the chain code

\subsection{Transformation of parameter with HMM}

A hidden Markov model (HMM) is collection of random variables consisting of a set of $\mathrm{T}$ discrete scalar variables Q1: $\mathrm{T}$ and a set of $\mathrm{T}$ other variablesX1: $\mathrm{T}$ which may be either discrete or continuous (and either scalar- or vector-valued) These variables, collectively, possess the following conditional independence properties:

$\{\mathrm{Qt:}$ T, Xt: T\}Џ $\{\mathrm{Q} 1: \mathrm{t}-2, \mathrm{X} 1: \mathrm{t}-1\} \mid \mathrm{Qt}-1$ 
And

$\mathrm{Xt} \biguplus\{\mathrm{Q} \neg \mathrm{t}, \mathrm{X} \neg \mathrm{t}\} \mid \mathrm{Qt})$

for each $t € 1: T$. No other conditional independence properties are true in general, unless they follow from above equations. The length $\mathrm{T}$ of these sequences is itself an integervalued random variable having a complex distribution.

It is going to determine by the supervised classification of chain code using HMM, which is the maximum rate of success, for extrapolating the forward and backward parameter of HMM in the calculation of Fisher score. Therefore, the HMM employed is a Bakis, and is trained with the procedure of Baum-Welch, to maximize the probabilities of success [3]. Besides, 8 symbols by state have been utilized. The creation of the HMM models has two phases, the training and the test. Finally, the number of states $(\mathrm{N})$ and the percentage of training samples have utilized like parameters to find the highest rate of success.

\subsection{Fisher score}

Finally, it is proposed the transformation that provides the HMM probabilities relating to the approach of the Fisher score [1]. With this goal, it intends to unite the probability given by the HMM to the given discrimination of the neural network, whose tie of union is this Fisher score. This score calculates the gradient with respect to the parameters of $\mathrm{HMM}$, in particular, on the probabilities of emission of a vector of data $x$, while it is found in a certain state $q \in$ $\{1, \ldots \ldots ., N\}$, given by the matrix of symbol probability in state $\mathrm{q}\left(\mathrm{b}_{\mathrm{q}}(\mathrm{x})\right)$, just as it is indicated in the following equation [1][8];

$\mathrm{P}(\mathrm{x} / \mathrm{q}, \lambda)=\mathrm{b}_{\mathrm{q}}(\mathrm{x})$

If it is realized the derivate of the logarithm of the above probability, with the purpose to calculate its gradient, it is obtained the kernel of Fisher, whose expression comes given by [1][8];

$$
\partial \log \mathrm{p}(\mathrm{x} / \mathrm{q}, \lambda) / \partial \mathrm{p}(\mathrm{x}, \mathrm{q})=\zeta(\mathrm{x}, \mathrm{q}) / \mathrm{b}_{\mathrm{q}}(\mathrm{x})-\zeta(\mathrm{q})
$$

where in [1], it has been found the approximations and the calculation of above equation. Besides, $\zeta(x, q)$ represents the number of times, that is localized in a state $q$, during the generation of a sequence, emitting a certain symbol $\mathrm{x}$ [1][3]. And $\zeta(q)$ represents the number of times that has been in $\mathrm{q}$ during the process of generation of the sequence [1][3]. These values are obtained directly and of form effective, from the forward backward algorithm, applied to the HMM [3] [8]. The application of this score $\left(\mathrm{U}_{\mathrm{x}}\right)$ to the neural network, comes given by the expression of the equation 2, utilizing the techniques of the natural gradient, from the following equation [2];

$\mathrm{U}_{\mathrm{x}}=\nabla_{\mathrm{p}(\mathrm{x}, \mathrm{q})} \log \mathrm{p}(\mathrm{x} / \mathrm{q}, \lambda)$

where $U_{x}$ define the direction of maximum slope of the logarithm of the probability to have a certain symbol in a state.

\section{NEURAL NETWORK (NN)}

The objective of this study is to classifying Fisher kernel data of sign language number symbol using feed forward back propagation neural network and Levenberg-Marquardt (LM) as the training algorithm. In this paper, LM training algorithm is adopted for updating each connection weights of units. LM algorithm has been used in this study due to the reason that the training process converges quickly as the solution is approached. For this study, sigmoid, hyperbolic tangent functions are applied in the learning process. Feed forward back propagation neural network use to classify sign language number symbol according to fisher score characteristic. Feed forward back propagation neural network is created by generalizing the gradient descent with momentum weight and bias learning rule to multiple layer networks and nonlinear differentiable transfer functions. Input vectors and the corresponding target vectors are used to train feed forward back propagation neural network. Neural network train until it can classify the defined pattern. The training algorithms use the gradient of the performance function to determine how to adjust the weights to minimize performance. The gradient is determined using a technique called back propagation, which involves performing computations backwards through the network. The back propagation computation is derived using the chain rule of calculus. In addition, the transfer functions of hidden and output layers are tan-sigmoid and tan-sigmoid, respectively.

\section{Training and Testing:}

The proposed network was trained with fisher score data cases. When the training process is completed for the training data, the last weights of the network were saved to be ready for the testing procedure. The time needed to train the training datasets was approximately 4.60 minutes. The testing process is done for 500 cases. These 500 cases are fed to the proposed network and their output is recorded.

Performance plot: Performance plot show the training errors, validation errors, and test errors appears, as shown in the training process. Training errors, validation errors, and test errors appears, as shown in the following figure 8.

Receiver Operator Characteristic Measure (ROC)

Plot: The colored lines in each axis represent the ROC curves. The ROC curve is a plot of the true positive rate (sensitivity) versus the false positive rate (1 -specificity) as the threshold is varied. A perfect test would show points in the upper-left corner, with $100 \%$ sensitivity and $100 \%$ specificity. For this problem, the network performs very well. The results show very good quality in the following figure 9 .

Regression plots: This is used to validate the network performance. The following regression plots display the network outputs with respect to targets for training, validation, and test sets. For a perfect fit, the data should fall along a 45 degree line, where the network outputs are equal to the targets. For this problem the fit is reasonably good for all data sets, with $\mathrm{R}$ values in each case of 0.93 or above. The results show in the following figure 10.

Training State Plot: Training state plot show the deferent training state in training process and validation check graph. These plots also show the momentum and gradient graph and state in training process. The results show in the following figure 11 .

\section{EXPERIMENTS AND RESULTS}

The outputs of hand sign image processing algorithms are depicted in the following figure 7 . The experiments have been realized with independent samples for training and for test. These have been repeated in five times to take averaged values, expressed them by their average and their variance. The execution of the same have been done sequential mode, first in the HMM to achieve its maximum success, varying the number of states and the percentage of samples in the training. 
The experimental results when varying the number of states and states of HMM are shown in table 1. As a result, the recognition accuracy was improved by increasing the number of states and the highest recognition accuracy was obtained in 65 states. Although the recognition accuracy was hardly improved when the number of samples increased, the highest recognition accuracy was obtained in $80 \%$ samples. As a result, recognition accuracy was $92.50 \%$ by using only the position of hands. The results are the following:

\begin{tabular}{|c|l|l|l|l|l|}
\hline \multirow{2}{*}{$\begin{array}{c}\text { Samples } \\
\text { Training }\end{array}$} & \multicolumn{5}{|c|}{ Number of state } \\
\cline { 2 - 6 } & 20 & 45 & 45 & 65 & 100 \\
\hline $20 \%$ & $73.66 \%$ & $84.60 \%$ & $85.74 \%$ & $84.38 \%$ & $62.40 \%$ \\
& \pm 22.82 & \pm 3.33 & \pm 2.47 & \pm 7.67 & \pm 45.65 \\
\hline $40 \%$ & $76.20 \%$ & $86.70 \%$ & $88.50 \%$ & $90.10 \%$ & $70.20 \%$ \\
& \pm 12.83 & \pm 3.50 & \pm 7.24 & \pm 1.82 & \pm 26.50 \\
\hline $60 \%$ & $74.78 \%$ & $89.90 \%$ & $87.30 \%$ & $86.98 \%$ & $65.10 \%$ \\
& \pm 11.20 & \pm 2.95 & \pm 3.90 & \pm 3.10 & \pm 25.87 \\
\hline $80 \%$ & $75.31 \%$ & $90.75 \%$ & $91.20 \%$ & $92.50 \%$ & $71.65 \%$ \\
& \pm 31.42 & \pm 14.19 & \pm 8.30 & \pm 14.15 & \pm 16.80 \\
\hline
\end{tabular}

Table 1: Rate of success of the HMM in function of the percentage of samples for training and of the number of states

Of the above table is deduced that the best percentage of training is fur $80 \%$ and 65 states, with a rate of $92.50 \%$, presenting a small variance. Of this model is going to generate the kernel of Fisher, and it is going to apply to the neural network. The results of these tests are collected in the following table 1. It is observed as for the neural networks are presented better results, arriving to an average rate of $98.34 \%$, with the smallest variance. The overall accuracy of sign language recognition in the training, validation and testing mode are $99.60,98.64$ and $98.52 \%$. We are concluding that that the proposed system gives fast and accurate sign language recognition. Given the encouraging test results, we are confident that an automatic sign language recognition system can be developed.

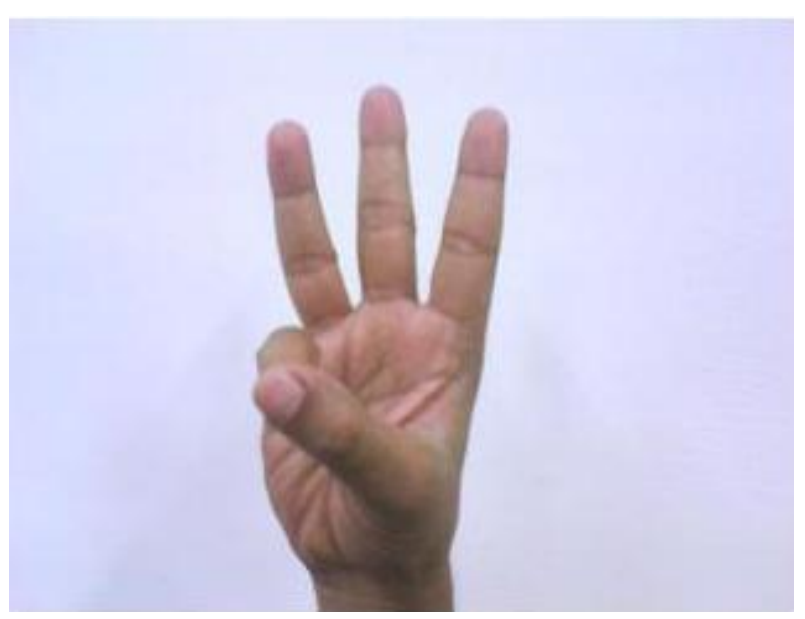

(1) Original Image

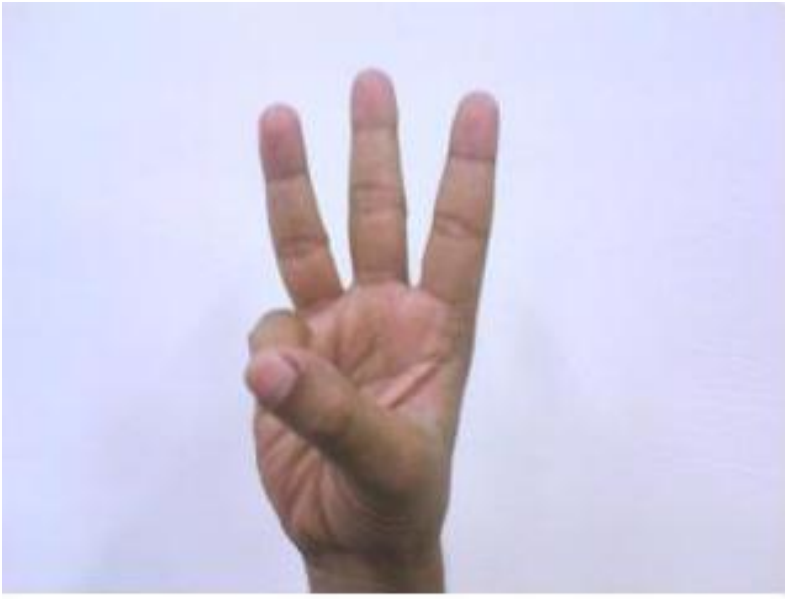

(2) Resize Image

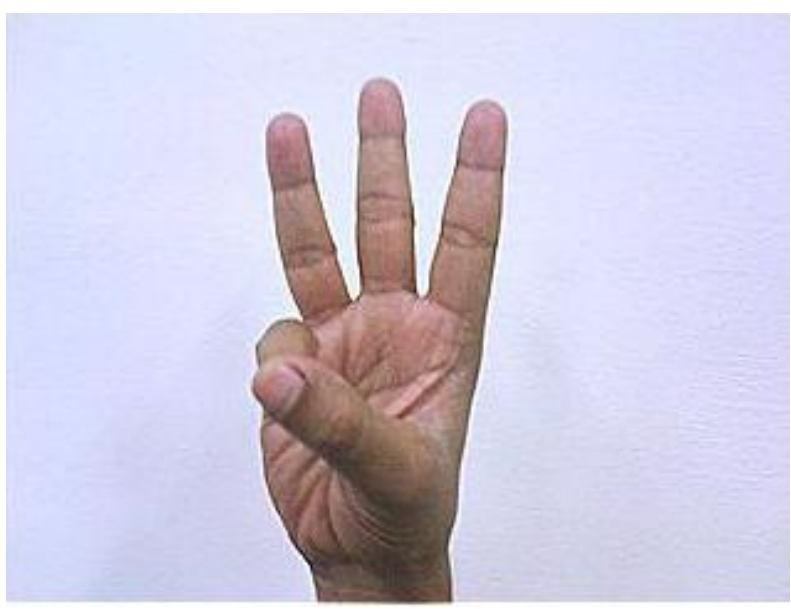

(3) Filter Image

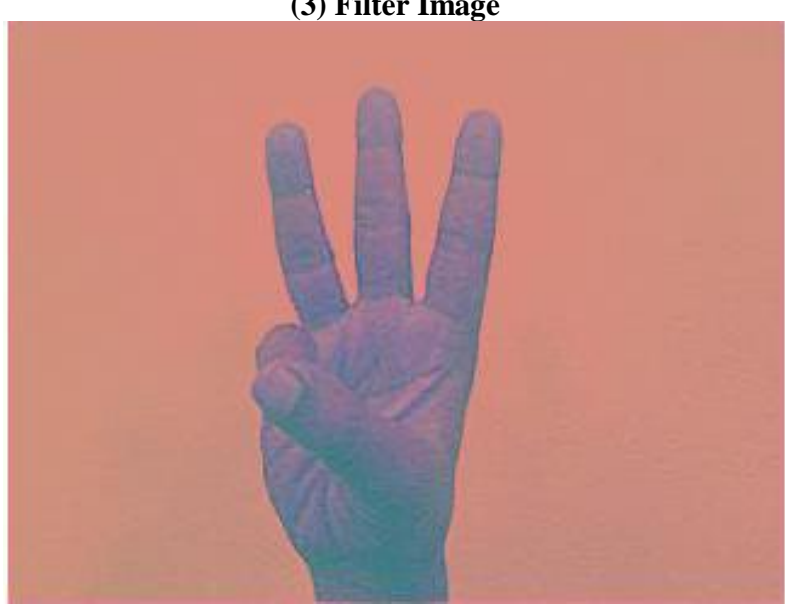

(4) YCBCR Color Image 


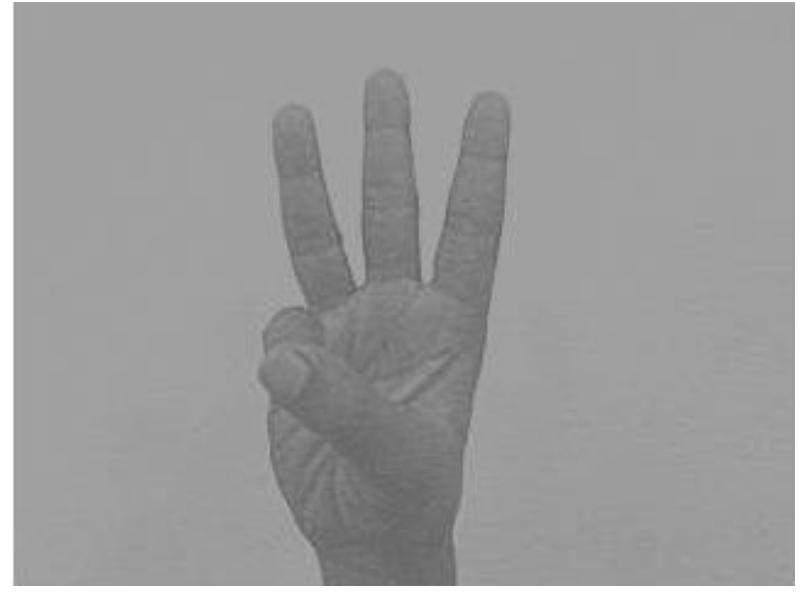

(5) Gray Image

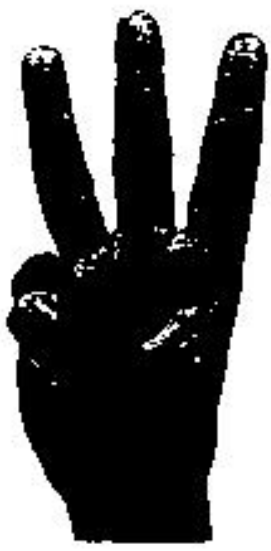

(6) Binary Image

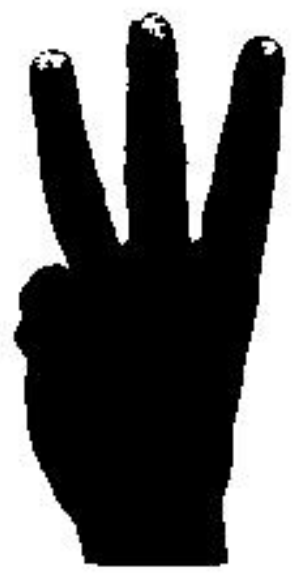

(7) Morphologic operation

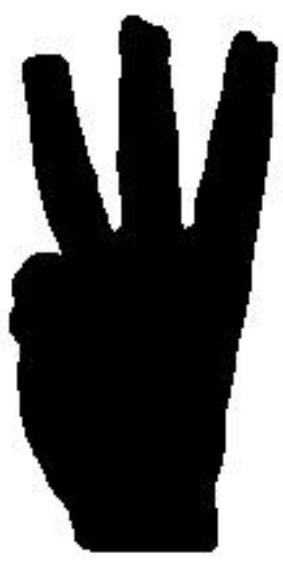

(8) Close operation

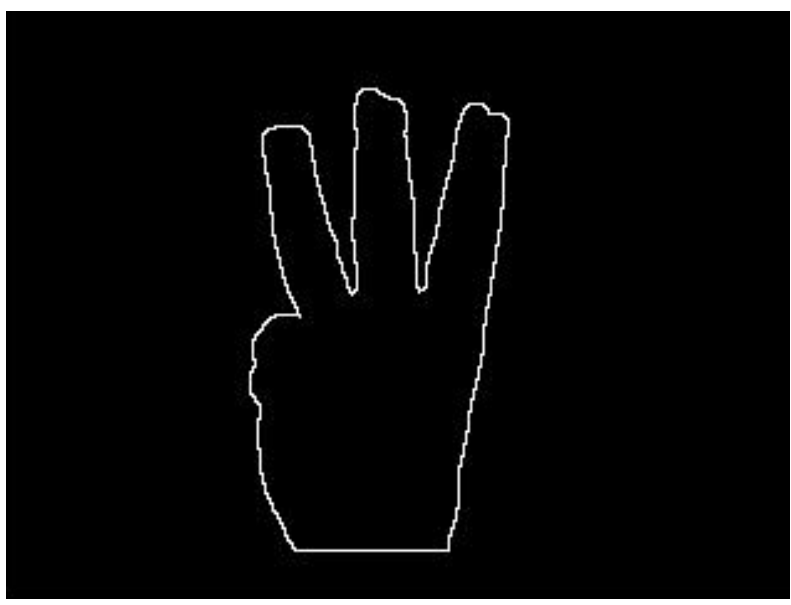

(9) Outline of image

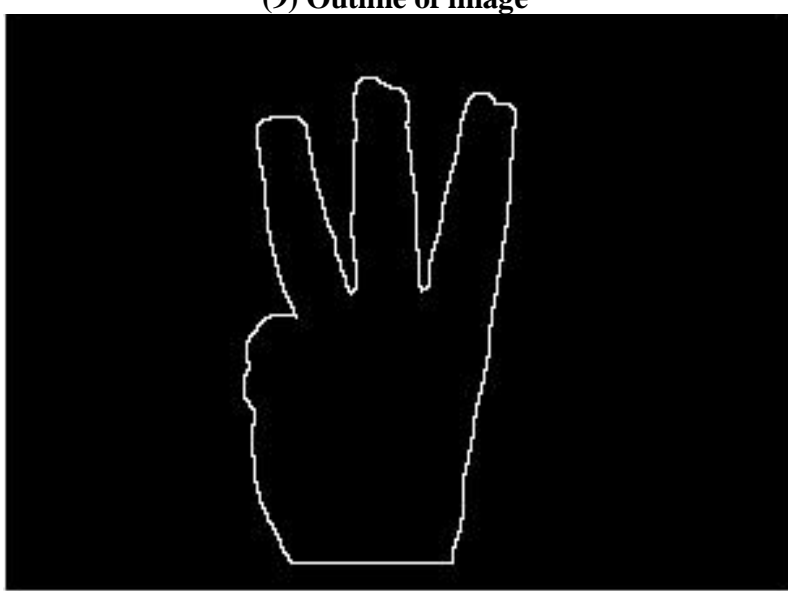

(10) Crop Image 


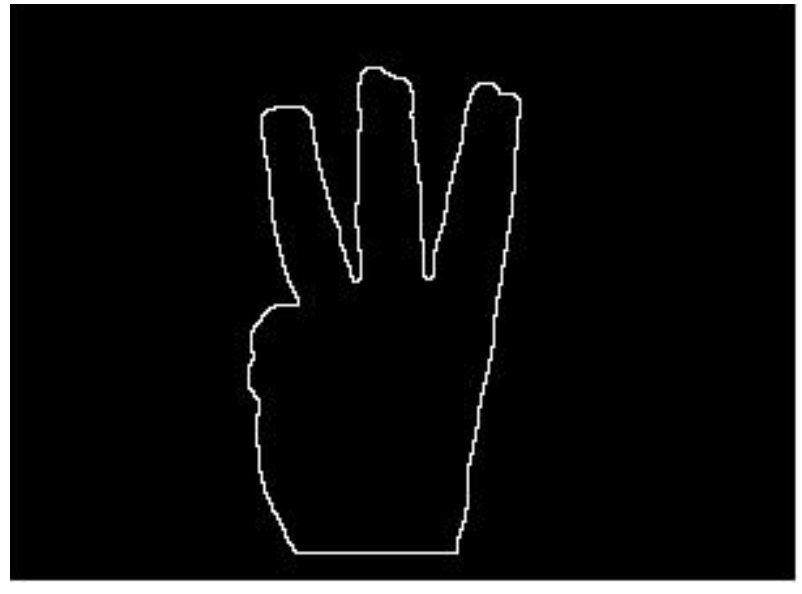

(11) Adjustment of image

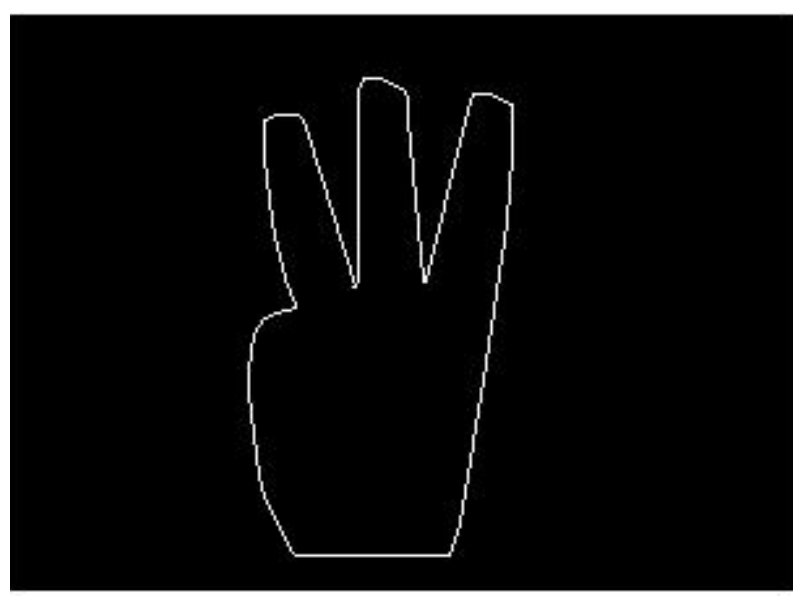

(12) Minimum polygon adjustment

Figure 7: hand sign image processing results

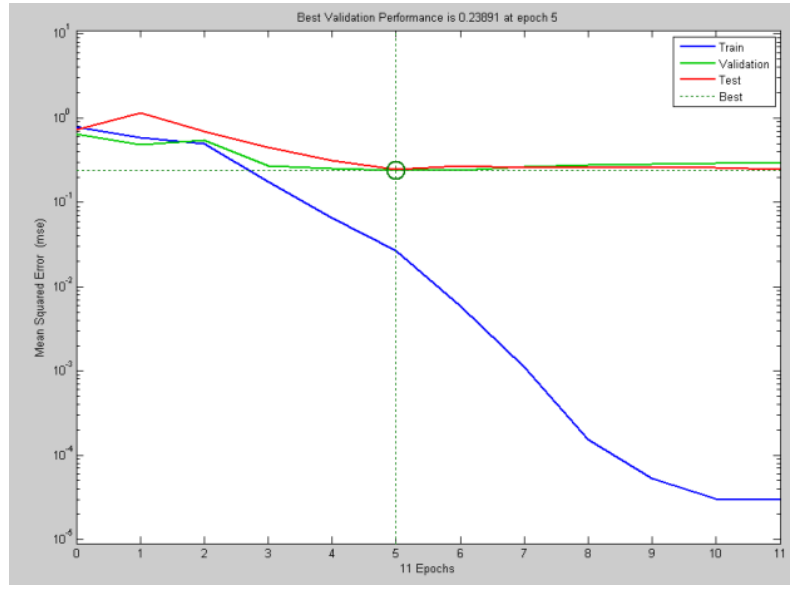

Figure 8: Performance plot

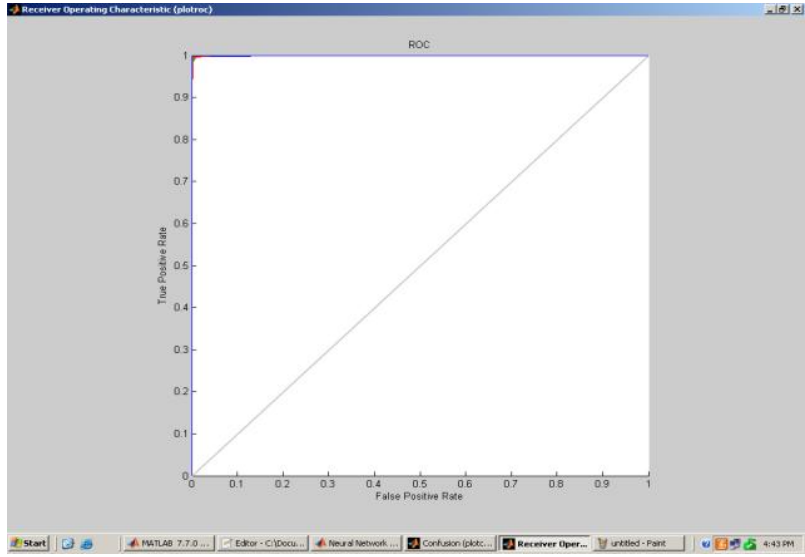

Figure 9: ROC plot

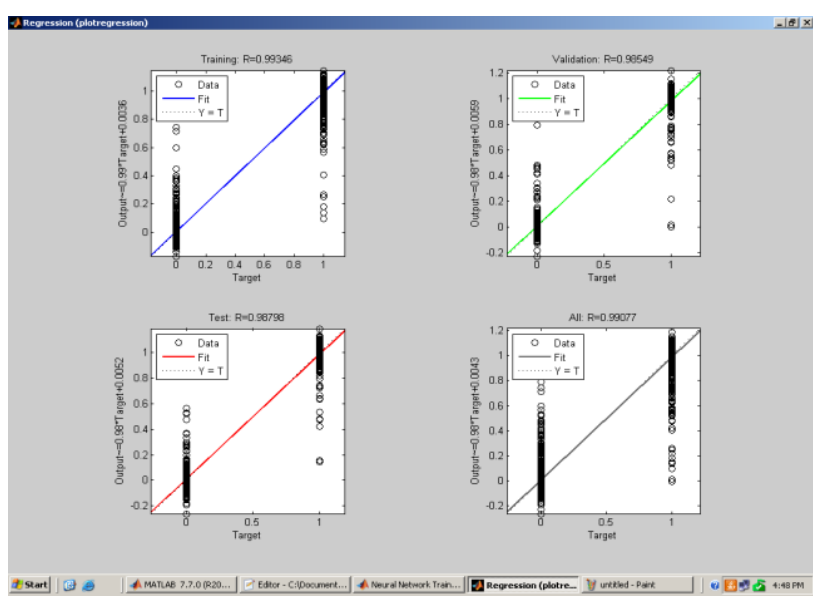

Figure 10: Regression plot

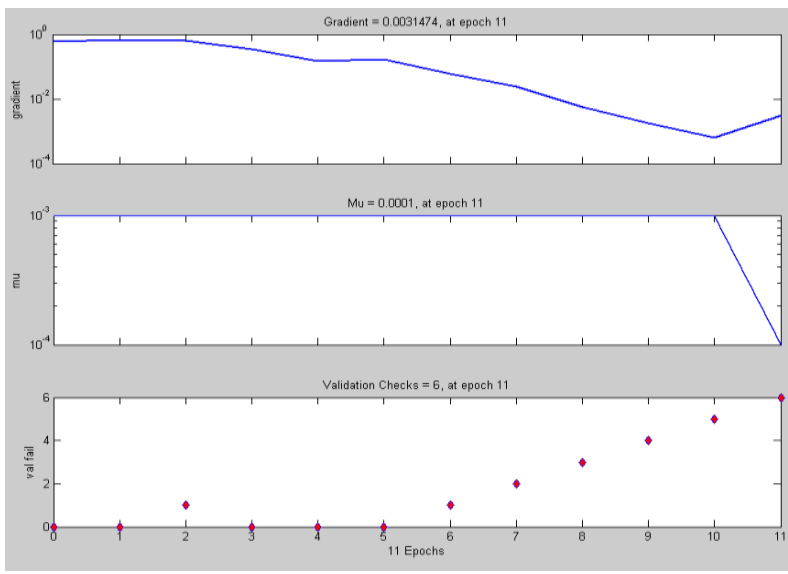

Figure 11: Training State Plot 


\section{CONCLUSION}

Indian Sign language number recognition system in this research was able to design a model for recognizing sign language number that was suitable for number in Indian Sign Language. The sign language number recognition system was also evaluated in terms of accuracy and time. The 65-state HMM without skip state has the highest average accuracy which is $95.32 \%$.The total average running time for 65 -state HMM was 42 minutes. In this article a robust and novel automatic sign language recognition system has been presented, from the transformation of chain code of the outline by HMM until Fisher score, related to the forward backward probabilities into HMMs. The overall accuracy of sign language recognition in the training, validation and testing mode are $99.60,98.64$ and $98.52 \%$. We are concluding that that the proposed system gives fast and accurate sign language recognition. Given the encouraging test results, we are confident that an automatic sign language recognition system can be developed.

\section{REFERENCES}

[1] Jaakola, T., Diekhans, M., and Haussler, D. (1998). "A discriminative framework for detecting remote protein homologies". Unpublished, available from http://w.cse.ucsc.edu/research/comphio/research.htnd.

[2] Christopher J. C. Burges, "A Tutorial on Support Vector Machines for Pattern Recognition", Data Mining and Knowledge Discovery, vol. 2, number 2, pp, 121-167, 1998.

[3] Lawence R. Rabiner, "A tutorial on Hidden Markov models and Selected Applications in Speech Recognition", in Proceedings of the IEEE, vol. 77, no. 2, pp. 257-286, 1989.

[4] L. O'Gorman and R. Kasturi, Document Image AnalJsis, IEEE Computer Society Press, 1995.
[5] J. Serra, Image Analysis and Mathematical Morphology, Academic Press, 1982

[6] C. Travieso, C. Morales, I. Alonso y M. Ferrer, "Handwritten digits parameterisation for HMM based recognition", Proceedings of the Image Processing and its Applications,vol.2, pp. 770-774, julio de 1999.

[7] E. Gomez, C.M. Travieso, J.C. Briceiio, M.A. Ferrer, "Biometric Identification Svstem by Lip Shape", in Proceeding of $361 \mathrm{~h}$ International Carnahan Conference on Security Technology, Atlantic City, October 2002,pp. $39-42$.

[8] Carlos M. Travieso, Jesus B. Alonso, Miguel A. Ferrer,"Sign language to text by SVM", 0-7803-79462/03, IEEE , 2003.

[9] Zhang, L.-G., Chen, Y., Fang, G., Chen, X., \& Gao, W. (2004). A vision-based sign language recognition system using tied-mixture density hmm. In ICMI '04: Proceedings of the 6th international conference on Multimodal interfaces, pages 198-204, New York, NY, USA. ACM.

[10] Wang, Q., Chen, X., Zhang, L.-G., Wang, C., \& Gao, W. (2007). Viewpoint invariant sign language recognition. Computer Vision and Image Understanding, 108:87-97.

[11] Starner, T., Weaver, J., \& Pentland, A. (1998). Real-time American sign language recognition using desk and wearable computer based video. Transactions on Pattern Analysis and Machine Intelligence, 20(12):1371-1375.

[12] Vogler, C. \& Metaxas, D. (2004). Handshapes and movements: Multiple-channel ASL recognition. In Springer Lecture Notes in Artificial Intelligence. Proceedings of the Gesture Workshop'03, Genova, Italy. pages 247-258. 